\title{
Quaderni
}

QUADERNI Communication, technologies, pouvoir

79 | Automne 2012

Produire la démocratie

\section{Des sciences de la participation : paysage participatif et marché des biens savants en France}

Cécile Blatrix

\section{OpenEdition}

Journals

Édition électronique

URL : http://journals.openedition.org/quaderni/612

DOI : 10.4000/quaderni.612

ISSN : 2105-2956

Éditeur

Les éditions de la Maison des sciences de l'Homme

Édition imprimée

Date de publication : 5 octobre 2012

Pagination : 59-80

Référence électronique

Cécile Blatrix, «Des sciences de la participation : paysage participatif et marché des biens savants en France », Quaderni [En ligne], 79 | Automne 2012, mis en ligne le 05 octobre 2014, consulté le 30 avril 2019. URL : http://journals.openedition.org/quaderni/612 ; DOI : 10.4000/quaderni.612 


\section{$D$ ossier}

\section{Des sciences de la participation :}

paysage participatif et marché des biens savants en France

\section{Cécile Blatrix}

Enseignante-chercheuse en science politique, AgroParisTech,

Centre Européen de Sociologie et de Science Politique (CESSP)
"Les chercheurs sont aussi les militants des objets sur lesquels ils travaillent ». Tel était l'avertissement donné par l'historien et philosophe des sciences Dominique Pestre au panel, lors d'une conférence de citoyens sur les nanotechnologies organisée au début de l'année 2007 par le Conseil Régional d'Île-deFrance. C'est ce constat, formulé pour mettre en garde le panel quant aux discours experts sur les nanotechnologies, qui sert de point de départ à cet article. On se propose de montrer ici qu'il s'applique parfaitement à la recherche en sciences sociales sur la participation, thèse que l'on défendra en éclairant un angle mort de l'histoire sociale de la démocratie participative, à savoir la contribution spécifique des chercheurs. Dans le cas de la France, cette contribution propre des chercheurs au déploiement du paysage participatif français sous les formes qu'on lui connaît aujourd'hui, nous paraît tout à la fois centrale, et largement évacuée de l'analyse.

L'analyse de la genèse de la démocratie participative, et des conditions d'émergence et de consolidation des dispositifs participatifs reste, d'une façon générale, un point relativement aveugle $^{1}$ dans la littérature en sciences sociales ${ }^{2}$. Une telle analyse, restituant les formes de construction de la "demande sociale de participation » permet d' ' incarner » la genèse des dispositifs participatifs, en mettant en lumière les acteurs sans qui la « cause » participative n'existerait pas. Tout un travail collectif a été nécessaire pour faire de la "démocratie participative » un problème public légitime. Il n'existe pas une catégorie unique d'acteurs qui aurait fait sienne cette cause, mais bien plutôt un cortège improbable et hétérogène où l'on 
croise aussi bien des hauts-fonctionnaires, des militants associatifs mobilisés autour de conflits environnementaux, des mouvements sociaux (autogestionnaire, écologiste, altermondialiste, lanceurs d'alerte...), des élus locaux et nationaux, ainsi qu'un certain nombre d'experts et plus récemment de professionnels de la participation. La démocratie participative est ainsi devenue tout à la fois un marché et un champ professionnel ${ }^{3}$. Elle constitue un objet et un produit pour un espace émergent de professionnels de la participation dans différents univers sociaux (maitres d'ouvrages, consultants, élus, experts, fonction publique...). Comme dans de nombreux secteurs de l'action publique aujourd'hui, une caractéristique de cette coalition semble bien être que le Fonctionnaire, le Militant et le Savant se croisent et s'entrecroisent dans cet espace où l'Économique et le Politique sont étroitement imbriqués.

La contribution des chercheurs à la construction et à la consolidation de l'édifice participatif français sera analysée ici en combinant une approche diachronique et un point de vue synchronique. Le paysage participatif français a évolué dans le temps et s'est reconfiguré en intégrant partiellement les connaissances produites par les sciences sociales sur les dispositifs et leur fonctionnement. L'analyse de ces reconfigurations permet de comprendre pourquoi la contribution propre du champ scientifique au développement de la participation du public en France est à la fois centrale, et largement occultée. Cette implication des chercheurs peut prendre plusieurs formes, que l'on passera ensuite en revue en proposant une cartographie du champ des sciences de la participation tel qu'il se présente aujourd'hui, et une typologie en fonction du degré d'implication qu'elle traduit .

\section{Reconfigurations du paysage participatif et standardisation des cadres d'analyse}

Une première manière d'observer la contribution des chercheurs est d'identifier les grandes étapes de l'évolution des dispositifs et des paradigmes. Il existe un lien étroit entre la manière dont le paysage participatif se structure progressivement, et la production savante sur le sujet, qui nourrit et inspire constamment les pratiques, l'expérimentation et parfois l'institutionnalisation de nouveaux dispositifs ${ }^{4}$. Trois périodes peuvent être schématiquement distinguées dans l'émergence et l'institutionnalisation des dispositifs participatifs en France. À chacune de ces périodes correspond un modèle plus ou moins dominant de participation du public, ainsi qu'une configuration spécifique du rapport entre recherche et action publique et politique.

(1) Du début des années 1970 à la fin des années 1980: émergence d'espaces publics de débat et rareté relative des travaux de recherche. Jusqu'à la fin des années soixante, une conception stricte de la démocratie représentative prévaut, où la seule forme, à la fois nécessaire et suffisante, de participation du public est l'élection. Une association des acteurs concernés à l'élaboration des décisions existe à travers des arènes institutionnalisées et non publiques de négociation, mais de façon très inégale selon les secteurs d'action publique : c'est ce que Pierre Muller a bien montré à propos des politiques agricoles, et qu'il a nommé le «néo-corporatisme à la française $»^{5}$. 
À partir du début des années soixante-dix, et jusqu'à la fin des années quatre-vingt, on assiste à l'émergence progressive d'espaces publics de débat. Cette période est caractérisée par la rareté relative des dispositifs participatifs d'une part, et des travaux de recherche les concernant d'autre part. L'enquête publique préalable aux projets susceptibles d'affecter l'environnement et les concertations dans le domaine de l'urbanisme constituent les seuls moments où la consultation du public est obligatoire pour certains projets, à un stade assez avancé du processus de décision. L'essentiel des analyses relève alors du droit et notamment du droit de l'environnement : elles se centrent sur l'enquête publique et les procédures de concertation. De fait, de nombreux juristes en droit de l'environnement militent explicitement en faveur de la participation du public considérée comme une manière de défendre la prise en compte des préoccupations environnementales ${ }^{6}$. De son côté, la sociologie des organisations appliquée à l'analyse du système politico-administratif local met en évidence les phénomènes notabiliaires et la non-participation sur laquelle ils reposent. La démocratie participative est alors un discours de critique du pouvoir en place détenu par les notables cumulants.

L'institutionnalisation de dispositifs participatifs nouveaux va être rendue possible par la définition d'une conception de la « démocratie participative » conciliable avec la démocratie représentative, qui s'impose à l'issue de cette période. Pierre Grémion propose ainsi une définition de la « démocratie participative » qui entérine la conception selon laquelle elle est fondamentalement compatible avec la démocratie représentative, qu'elle ne fait que perfectionner : "La théorie de la démocratie participative ne cherche pas à modifier le régime représentatif lui-même (en particulier, elle ne préconise pas le mandat impératif); elle cherche à améliorer les mécanismes de délégation (sélection et renouvellement des leaders; intensité du lien représentant/représenté, etc.) en agissant sur les mécanismes de fonctionnement des institutions $»^{7}$. À cette époque, l'expertise en matière de participation du public est peu structurée. Aucun des grands corps techniques ou administratifs ne dispose alors de l'expertise nécessaire en la matière. Rappelons qu'après la seconde Guerre mondiale, et plus précisément sous la $V^{\mathbb{R}}$ République, le modèle de l'expertise, importé des États-Unis et faisant largement appel aux sciences sociales, avec la mise en place d'une politique de planification ${ }^{8}$, n'intègre pas encore cette question. Ainsi, les grands programmes de recherche qui vont jouer un important rôle d'incitation et de coordination ne sont pas encore créés et ne verront le jour qu'à partir des années 90 .

(2) $1992-2002$ : Développement des dispositifs participatifs et d'une demande d'expertise. Les années 90 et le début des années 2000 constituent une phase de fort développement, voire de prolifération des dispositifs participatifs, et des travaux de recherche les concernant. Ainsi, aux enquêtes publiques et concertations en matière d'urbanisme, s'ajoutent les débats publics organisés par la Commission Nationale du Débat Public, les conseils de développement, conseils de quartiers, référendums locaux désormais encadrés par des textes juridiques 9 . Le débat public, expérimenté dès 1992 via la circulaire dite Bianco, est institutionnalisé pour la première 
fois par la loi Barnier en 1995, et la Commission Nationale du Débat Public verra ses compétences élargies par la loi démocratie de proximité en 2002. La demande de recherche et d'expertise en matière de participation émane alors de différents secteurs d'action publique, qui de façon concomitante ou successive, vont être demandeurs de savoirs spécifiques sur ce thème. Les politiques urbaines, politiques de la ville, politiques de l'environnement et de développement durable, politiques d'aménagement du territoire et de décentralisation sont autant de politiques publiques dans lesquelles des programmes de recherche ministériels font appel à une expertise dont les corps administratifs et techniques sont dépourvus. Des programmes de recherche et des appels d'offre apparaissent et contribuent au développement de ce champ de recherche, qui va lui-même alimenter la modernisation du processus de décision publique.

Ce sont surtout les administrations de l'Environnement et l'Équipement qui s'emparent du thème de la concertation dans les années 90 . Comme l'ont bien montré Marianne OllivierTrigalo et Xavier Piechaczyk, la rédaction de la charte de la concertation en 1995 illustre ainsi la manière dont deux logiques sectorielles concurrentes - portées par l'Environnement d'une part, et l'Équipement d'autre part - vont contribuer à l'institutionnalisation du débat public comme solution commune à un problème commun (décider mieux) ${ }^{10}$.

Du côté du ministère de l'Équipement, est mis en place dès 1990 le Programme de recherche et d'innovation dans les transports terrestres (PREDIT) dont la première vague (1990-1994) sera centrée sur les questions d'innovations techniques, mais qui à partir du PREDIT 2 (1996-2000) fait une large place aux sciences sociales. Mais c'est surtout le programme Concertation, Décision, Environnement (CDE), mis en place par le ministère de l'Écologie, qui constitue le premier programme centré sur la thématique participative ${ }^{11}$. Le premier appel à propositions de recherche de ce programme pluridisciplinaire en sciences sociales couvre la période 1999-2005. Des recherches portant sur les dispositifs institutionnalisés, mais aussi sur l'expérimentation de techniques nouvelles en dehors de tout cadre juridique seront ainsi financées dans le cadre de ce programme. C'est le cas, par exemple, de l'analyse des conférences de consensus conduites au Danemark, pour lesquelles une adaptation au contexte français est proposée, qui sera publiée dans un ouvrage en forme de «mode d'emploi ». Ce guide méthodologique fait aujourd'hui référence en la matière et est largement utilisé par les acteurs intéressés par l'utilisation de cette technique.

Ainsi les dispositifs qui se développent, qu'ils soient encadrés juridiquement ou non, sont promus par des ouvrages à caractère prescriptif, souvent présentés comme des guides méthodologiques, émanant de chercheurs et/ou d'associations militant pour la démocratie participative ou l'un des dispositifs censés l'incarner ${ }^{12}$.

Toujours à partir des années quatre-vingtdix, cette fois sur la base de l'émergence de politiques publiques locales en matière de participation rendues possibles par les politiques de décentralisation, un marché important se développe en provenance des autorités locales. 
Dans son étude de la genèse de la démocratie locale à travers l'institutionnalisation du référendum par la loi ATR en 1992, Marion Paoletti montre comment, alors que la décentralisation avait été revendiquée par les élus au nom de la nécessaire participation des habitants au niveau local, elle exclut dans un premier temps sa codification, au nom de la naturalité de cette participation dans la commune décentralisée ${ }^{13}$. L'affirmation du principe de sa compatibilité profonde avec la logique représentative qu'elle doit régénérer, rend possible l'institutionnalisation de procédures et de dispositifs, tout en vidant la notion de participation de son contenu potentiellement subversif. Le discours en faveur de la démocratie participative, jusqu'alors discours de critique du pouvoir en place porté par le milieu associatif, est repris à leur compte par les élus eux-mêmes, en tout cas certains d'entre eux, qui appellent de leurs vœux le développement de dispositifs de participation en dehors du vote.

Le marché qui se construit à cette époque présente une double caractéristique : il émane aussi bien des services administratifs et techniques que des élus locaux, dans des collectivités au positionnement politique souvent clairement ancré à gauche $(\mathrm{PS}, \text { Verts, } \mathrm{PCF})^{14}$. Il s'agit d'un marché relativement territorialisé, où les liens sont tissés de façon privilégiée avec les institutions universitaires et de recherche ancrées dans le territoire $^{15}$. Ici l'expertise alimente directement les politiques locales en matière de participation, qu'elles aident à construire, à faire exister et à rendre plus visibles. Les politiques conduites en matière de recherche et les programmes de financement mis en place dans certains conseils régionaux (Île-de-France, Rhône-Alpes) vont renforcer cette logique à partir des élections régionales de 2004. L'offre d'expertise s'adresse en effet à un troisième type de clientèle, de façon moins visible, à savoir des organisations proprement politiques, qui sont marquées sous la $V^{e}$ République par le développement de leurs propres formes d'expertise à travers des clubs, commissions, demandes d'experts. C'est le cas des organisations syndicales, des partis politiques, de certaines structures associatives (FNE, ADELS...).

\section{(3) 2002-2012: normalisation d'une approche} normative et autonomisation d'un champ de recherche. Au début des années 2000, un paradigme délibératif semble s'emparer de la recherche sur la participation ${ }^{16}$. Le constat du développement d'un impératif participatif que nous formulions en 2000 est requalifié en termes d' « impératif délibératif » en 2002, avec une ambiguïté quant à la nature de ce «tournant» : en réalité il s'agit autant d'un tournant dans les cadres théoriques retenus, que dans les réalités observées des pratiques de participation. C'est en effet largement sous l'impulsion de cette orientation résolument délibérative des cadres théoriques, inspirés à la fois par les travaux de J. Habermas et par les théories de la démocratie délibérative développées aux États-Unis, que certains dispositifs vont être portés au premier plan dans la hiérarchie des objets légitimes de la recherche sur la démocratie participative.

Le « tournant délibératif » est ainsi marqué par un relatif désintérêt pour certaines procédures et certaines questions, comme l'analyse empirique de la sociologie des participants. Celle-ci montrant que la participation aux dispositifs participatifs 
est caractérisée par les mêmes inégalités sociales que la particpation électorale, avec de surcroît une vaste majorité de "non-participants $»^{17}$, on va privilégier, à travers une approche indissociablement descriptive et prescriptive, les procédures et les formes de construction du (mini) public idéal, que ce soit en faisant appel à des instituts de sondage, ou en réhabilitant le tirage au sort. Dans cette perspective, la conférence de citoyens (et assimilés) est au centre de toutes les attentions.

Tout se passe comme si, pour résoudre le problème que pose l'écart entre le fonctionnement pratique des dispositifs et le système de croyances et de valeurs qui les sous-tendent, se mettait en place une dynamique d'ingénierie procédurale conduisant à un glissement progressif de la participation comme moyen à la participation comme fin en soi.

Cette période est en effet marquée par une autre tendance, soulignée par plusieurs auteurs, où les sciences sociales consacrent, d'une certaine façon, les dispositifs de concertation comme le modèle de référence de l'action publique. Les sciences sociales entrent ainsi, selon le président $\mathrm{du}$ Conseil Scientifique du programme CDE, dans un « mouvement d'apologie de la recherche de l'accord $»^{18}$. On peut également, en reformulant légèrement le constat similaire fait par Bernard Manin en $2002^{19}$, parler d'une normalisation d'une approche normative dans les travaux sur la participation.

La réflexion en matière d'évaluation de la concertation est significative de cette tendance à considérer la participation comme une fin en $\mathrm{soi}^{20}$. La focalisation de l'attention de la recherche sur les questions de design institutionnel renvoie à un argument de la théorie de la justice procédurale selon lequel le respect de procédures équitables donne des résultats qui sont euxmêmes équitables ${ }^{21}$. D'où une quête sans fin de la « bonne » procédure et la mise en place d'un quasi réflexe consistant à ne pas évaluer les effets des dispositifs participatifs à l'aune de leurs objectifs annoncés $^{22}$. Ainsi les vertus prêtées à la démocratie participative et ayant servi à justifier la mise en place et l'institutionnalisation de nouveaux dispositifs, ne font l'objet d'aucune tentative d'évaluation. Lorsque des grilles d'évaluation sont proposées, elles sont centrées sur des questions de performance procédurale des dispositifs et laissent de côté les effets annoncés initialement, qu'il s'agisse des impacts sur la participation électorale, des effets en termes de légitimité des décisions et des décideurs, d'inclusion sociale et de lutte contre les inégalités, ou encore en termes de meilleure prise en compte de l'environnement ${ }^{23}$.

Une fois mis sur l'agenda et établi (sans être nécessairement codifié), un dispositif participatif peut être ainsi étudié et reconfiguré à l'infini, afin d'améliorer toujours plus sa (seule) performance procédurale ${ }^{24}$.

Si les programmes de recherche évoqués précédemment continuent à exister, la participation tend à s'autonomiser en tant que champ de recherche spécifique, avec une revue dédiée, des formations, et, aboutissement de cette logique, une structuration au sein d'un Groupement d'Intérêt Scientifique créé en 2008. Contrairement à d'autres GIS, le GIS Démocratie 
et participation ne finance pas de recherche. Financé par ses « organismes partenaires $»^{25}$ (organismes de recherche, opérateurs, associations d'élus et institutions concernées ou directement en charge de la participation du public), il vise à fédérer et coordonner la recherche sur cette thématique. Comme indiqué sur son site internet, le GIS «constitue ainsi l'outil privilégié de dialogue sur ces recherches entre la communauté des chercheurs en sciences humaines et sociales (notamment dans les organismes partenaires $d u$ GIS : CNRS, IFSTTAR, IRSTEA, CIRAD, INRA), les institutions et entreprises ayant en charge la mise en ouvre de la participation du public aux processus décisionnels (notamment celles qui sont partenaires du GIS : ADEME, IRSN, Association des Régions de France, Commission nationale du débat public, Réseau ferré de France, Réseau de transport d'électricité, EDF), et la société civile ».

Le tableau (1) suivant montre que chaque période que nous venons de décrire peut être schématiquement caractérisée par un modèle dominant de participation, ce qui ne signifie pas, bien sûr, que les nouveaux formats abolissent

\begin{tabular}{|c|c|c|c|}
\hline & $\begin{array}{l}\text { Format dominant de } \\
\text { participation }\end{array}$ & $\begin{array}{l}\text { Principales difficultés } \\
\text { révélées par les recherches }\end{array}$ & \multirow[b]{2}{*}{$\begin{array}{l}\text { Impératif } \\
\text { Participatif }\end{array}$} \\
\hline $\begin{array}{l}\text { Sphères } \\
\text { institutionnalisées } \\
\text { de négociation }\end{array}$ & $\begin{array}{l}\text { Comités de bassin } \\
\text { Lieux institutionnalisés et non } \\
\text { publics de négociation entre } \\
\text { parties prenantes }\end{array}$ & $\begin{array}{l}\text { Manque de transparence, } \\
\text { absence de mécanisme de } \\
\text { contrôle }\end{array}$ & \\
\hline $\begin{array}{l}\text { Sphères } \\
\text { publiques de }\end{array}$ & $\begin{array}{l}\text { Débat public (CND) } \\
\text { Moments publics et ouverts } \\
\text { (réunions publiques); aucune } \\
\text { restriction à la participation }\end{array}$ & \multicolumn{2}{|l|}{$\begin{array}{l}\text { Public composé principalement } \\
\text { de «représentants de fait» } \\
\text { Restrictions pratiques de la } \\
\text { participation, notamment du fait } \\
\text { de son ancrage territorial et de } \\
\text { biais procéduraux }\end{array}$} \\
\hline & & $\begin{array}{l}\text { Difficulté à mobiliser certaines } \\
\text { catégories de population } \\
\text { (jeunes, démunis...) }\end{array}$ & \multirow[t]{2}{*}{$\begin{array}{l}\text { Impératif } \\
\text { Délibératif }\end{array}$} \\
\hline $\begin{array}{l}\text { Délibération en } \\
\text { petits groupes }\end{array}$ & $\begin{array}{l}\text { Conférences de citoyens } \\
\text { construisant des représentants } \\
\text { de la diversité des citoyens } \\
\text { ordinaires } \\
\text { (parfois considérés comme } \\
\text { des représentants des } \\
\text { générations futures) }\end{array}$ & $\begin{array}{l}\text { Petit nombre de participants ; } \\
\text { forte logique d'apprentissage } \\
\text { pour ces participants } \\
\text { Impacts limités au-delà du } \\
\text { panel même si ces expériences } \\
\text { se multiplient }\end{array}$ & \\
\hline
\end{tabular}


ceux qui leur préexistent.

Chaque nouvelle étape franchie est alimentée par l'analyse faite par la recherche en sciences sociales du fonctionnement pratique des dispositifs et de leurs limites, analyse qui contribue à générer une réflexion permettant le renouvellement de l'ingénierie procédurale.

Ainsi, le constat du caractère inégalement public des débats publics, qui sont investis de manière très différenciée selon les catégories d'acteurs et qui ne parviennent pas à mobiliser le « grand public », va ainsi largement nourrir la réflexion et l'expérimentation en vue de formes d'ingénieries mettant l'accent sur les techniques permettant d'atteindre une haute qualité délibérative des échanges plutôt qu'une large participation du public. Après l'expérimentation pour la première fois en France en 1998, de la conférence de citoyens, sur l'utilisation des OGM dans l'agriculture, ce dispositif se répand avec de nombreuses expériences, non seulement à l'échelon national mais de plus en plus à l'échelon local, à l'initiative aussi bien d'entreprises privées que de collectivités territoriales.

Un nouveau marché se développe ainsi pour l'expertise en matière de construction / consultation de « mini-publics ». Certains dispositifs peuvent ainsi paraître directement inspirés de la volonté de tester empiriquement des propositions et hypothèses issues de théories qui se diffusent progressivement et imprègnent les agendas politiques. Demande politique, innovations institutionnelles et interrogations théoriques sont fortement liées ${ }^{26}$. Les dispositifs participatifs tirent donc en partie leur origine de la diffusion de préoccupations de la recherche, qui leur fournit, au moment opportun, une légitimité, souvent sous la forme de règles de bonne conduite dont le respect est dûment attesté par des tiers dont les chercheurs font partie.

Les reconfigurations successives de cet ensemble hétérogène d' « innovations » que recouvre l'idée de démocratie participative et/ou délibérative, doivent donc beaucoup à la contribution du champ académique. Ceci permet d'élucider l'énigme de la perpétuation d'un ensemble de « solutions » dont on sait pourtant qu'elles ne règlent aucun des problèmes qu'elles prétendent adresser. L'analyse de la dynamique de la construction sociale de la démocratie participative, et du rôle de la recherche dans cette dynamique, permet de saisir une des logiques de son fonctionnement. Même si son importance ne doit pas être surestimée par rapport à d'autres facteurs, la recherche constitue bien un rouage essentiel de ce processus et la prise en compte de ce rôle permet de comprendre le déploiement du paysage participatif français et ses évolutions, ainsi que la capacité des dispositifs participatifs (certains plus que d'autres) à circuler et essaimer. Mais la contribution de la recherche à la construction de la démocratie participative doit aussi être saisie de façon synchronique pour en appréhender l'organisation et les différentes facettes.

\section{La construction d'un marché des biens savants. Positions et prises de position des sciences de la participation}

Nous allons à présent tenter de dégager plus précisément les formes que cette contribution peut prendre et de représenter graphiquement 
la manière dont ce champ est structuré. La production scientifique sur la démocratie participative peut s'analyser en termes de construction d'un marché des biens savants ${ }^{27}$. Diffusion des «innovations », travail de normalisation, et processus de structuration de ce marché28 semblent se conjuguer aujourd'hui pour donner naissance à une dynamique de normalisation assez particulière, puisqu'il s'agit essentiellement d'une normalisation en termes de « libre conformation».

La contribution de la recherche sur la participation à la construction de l'objet qu'elle se propose d'analyser peut prendre des formes très diverses, que l'on distinguera ici à des fins d'exposition mais qui se recouvrent et s'imbriquent largement. Les cinq types de contributions sont exposés selon leur gradation sur une échelle de la participation de la recherche à la construction de la démocratie participative - clin d'œil à l'échelle élaborée en 1969 par S. Arnstein.

\section{Des porte-parole d'une "demande sociale de papier "}

La recherche peut contribuer à faire exister la « demande sociale » qui permet de justifier l'offre de participation, soit jouer un rôle plus direct dans la mise sur l'agenda d'un problème pour lequel la participation semble constituer une solution, même partielle et provisoire. De façon diffuse, les chercheurs contribuent ainsi, parmi les nombreux autres acteurs évoqués en introduction, à accréditer l'idée d'une demande sociale de participation sans questionner sa réalité, ses conditions de production son caractère inégalement réparti.
L'univers académique peut aussi promouvoir le développement de dispositifs de concertation comme moyen d'atteindre un objectif plus spécifique. Le cas de l'environnement fournit de nombreux exemples de ce type. Les travaux et préconisations portés par toute une génération de juristes en droit de l'environnement ont sans doute joué un rôle majeur dans l'institutionnalisation progressive de la concertation, à travers les réformes successives de l'enquête publique ou encore tout récemment, la mise en place au sein de la Loi Grenelle 2 d'une obligation de mise en ligne des projets de textes réglementaires nationaux, sur le modèle de la procédure anglosaxonne du Notice and Comment ${ }^{29}$. Dans d'autres cas, mues par des préoccupations intellectuelles spécifiques, les sciences sociales en général et la science politique en particulier, ont pu contribuer à la formulation d'injonctions plus ou moins explicites en direction du champ politique, en faveur de l'expérimentation de formes novatrices de consultation. Ce faisant, elles alimentent les débats précédant les réformes et les innovations participatives. Il y a également de fortes chances pour que les chercheurs soient ensuite les principaux défenseurs de ces expériences qu'ils auront activement contribué à créer ${ }^{30}$.

\section{Des chercheurs " catalyseurs " : circulation internationale des dispositifs et marketing démocratique territorial}

Le deuxième type de contribution spécifique de la recherche en participation à la construction de son objet renvoie à la diffusion des pratiques participatives présentées comme des innovations démocratiques, cette fois par la recherche spécialisée sur ces objets. Le terme de diffusion 
renvoie ici à la fois à l'idée d'une dissémination et à celle d'une promotion des dispositifs. Cette promotion n'est pas pensée comme telle, et constitue surtout un effet de la mise à disposition d'informations et de connaissances sur les pratiques participatives dans l'espace public qui résulte du travail des chercheurs.

La diffusion des innovations démocratiques que constituent les dispositifs de participation obéit à des logiques largement étudiées par une tradition de recherche abondante, issue de disciplines variées, qui s'intéresse à l'émergence et à la diffusion des innovations et qui examine les processus par lesquels de nouvelles idées, pratiques, et de nouveaux objets se répandent. Ces innovations n'ont pas besoin d'être objectivement « nouvelles ». Il faut seulement qu'elles soient perçues comme telles par un acteur susceptible de les faire siennes. Selon ces travaux, la question des sources et ressources, sur lesquelles les décideurs politiques s'appuient pour obtenir les informations dont ils ont besoin, est décisive pour comprendre le rôle croissant des acteurs les plus aptes à proposer des informations adaptées aux contraintes (de divers ordres, mais notamment de temps), de l'action politique ${ }^{31}$. En matière de diffusion des innovations politiques, il apparaît que les élus ont, peut-être encore plus que d'autres acteurs, tendance à s'appuyer sur l'information qui est rapidement disponible à moindre coût et pertinente (dit autrement, rendue visible et mise à disposition au bon endroit au bon moment). L'heuristique de disponibilité suggère ainsi que des exemples hautement visibles peuvent alors servir de raccourcis pour se procurer de l'information ${ }^{32}$.
On peut distinguer deux formes différentes - qui dans la réalité sont souvent étroitement imbriquées - que peut prendre ce rôle de diffusion des dispositifs, qui se manifeste aussi bien à travers la circulation internationale des idées et des dispositifs, qu'à travers un marketing démocratique territorial.

La recherche spécialisée met à disposition des connaissances sur une nouvelle pratique, ses modalités d'organisation, les conditions auxquelles elle pourra être transférée et adaptée à des contextes variés. L'expertise en participation est ainsi à même de proposer des prestations permettant de diminuer les coûts de la recherche d'information. Elle peut éventuellement contribuer à rendre ces innovations plus accessibles, en quelque sorte en démocratisant l'accès aux technologies de participation, par sa capacité à la configurer selon un format adapté aux contraintes de temps et de budget des acteurs.

Elle autorise ainsi des profits liés à la visibilité et à la forme de reconnaissance qu'elle donne à ces pratiques. La pluralité des espaces (économique, politique, administratif, académique...) dans lesquels les experts en participation naviguent, garantit une visibilité et une notoriété à toute expérience locale qui saura capter leur attention ou financer une recherche.

Pour que ce rôle de promotion puisse jouer à plein, il importe de rendre disponibles ces technologies, dont les experts en participation professent la neutralité politique a priori. La visibilité donnée à une pratique locale peut donc être sans aucune proportion avec sa capacité à atteindre les objectifs annoncés. Elle est liée au rôle de 
catalyseur joué par l'ensemble des professionnels de la participation. On peut considérer que les chercheurs analysant les dispositifs participatifs ou théorisant les potentialités de la démocratie participative et/ou délibérative font eux aussi partie des «professionnels de la participation», et à ce titre contribuent à construire le phénomène dans la mesure où une partie d'entre eux n'hésite pas à endosser le rôle d'entrepreneurs de cause.

Ces acteurs, indique Magali Nonjon, sont amenés à jouer le rôle de catalyseur aux deux sens littéraux du terme, c'est-à-dire celui d'une substance qui augmente la vitesse d'une réaction chimique sans paraître participer à cette réaction, ou d'un élément qui provoque une réaction par sa seule présence ou son intervention. C'est ainsi que les professionnels de la participation, sans paraître participer aux politiques participatives, n'en contribuent pas moins à accroître leur force sociale et à façonner la demande de participation. Leur seule existence « a fini par constituer en soi un gage et une garantie que la démocratie participative est en marche $»^{33}$.

L'évocation de leurs pratiques et démarches participatives permet aux élus de se placer euxmêmes parmi l'avant-garde démocratique. Les règles propres à la concurrence politique poussent les élus à imiter ce que font leurs pairs, d'autant plus que les expériences participatives sont mises en avant, valorisées socialement et peu risquées politiquement. Les profits attachés au fait de « donner la parole » n'existent que dans la mesure où cette démarche vertueuse est mise en scène, ce dont ne se privent pas les élus se positionnant comme les plus avant-gardistes en la matière. Les politiques locales en matière de démocratie participative présentent des traits communs avec les politiques d'image analysées par Christian Le Bart $^{34}$. Le caractère indémontrable de leur (in)efficacité politique, le caractère improbable et même potentiellement « coûteux » de toute mobilisation qui consisterait à dénoncer la démarche visant à « donner la parole», semblent en particulier des caractéristiques importantes de ces politiques.

\section{Des chercheurs " déontologues " : formalisation d'une doctrine délibérative et garantie de son respect}

Le troisième type de contribution possible renvoie au rôle que des chercheurs vont jouer dans l'élaboration d'une doctrine, dotant une institution émergente d'une légitimité à la fois académique et démocratique. Il s'agit d'une forme de codification des comportements attendus des uns et des autres pour que la participation soit considérée comme "valable » et conforme aux attendus normatifs issus des théories et traditions d'analyse, sur lesquels les experts des sciences de la participation fondent leur autorité. Cette contribution à la consolidation d'un dispositif peut se faire à travers son institutionnalisation au sens de codification juridique : ce type de doctrine se retrouve aussi bien pour les conférences de citoyens (non inscrites dans notre droit) ${ }^{35}$ que pour le débat public (encadré par des textes depuis le début des années quatre-vingtdix). L'exemple des règles du débat public est significatif de ce mélange hybride de règles de bonne conduite (pour les participants), de règles déontologiques (pour les organisateurs et garants) et d'attendus normatifs. Ces règles du débat, qui décrivent et prescrivent tout à la fois le caractère 
nécessairement argumenté des échanges, et l'équivalence de principe des participants au débat public, ont été très largement et assez directement formulées par les chercheurs euxmêmes. On a ainsi pu observer « de l'intérieur» comment, autour de la réponse à un appel d'offres dans le cadre du programme PREDIT, ont été diffusés les concepts et idéaux habermassiens auprès de la CNDP alors en apprentissage. Contrairement à une idée répandue, et comme le rappelle d'ailleurs un ancien vice-président de la CNDP, l'idée de délibération n'a pas fondé la création de la $\mathrm{CNDP}^{36}$, mais intervient sous la forme d'une « mise en cohérence » a posteriori, une formalisation, une rationalisation, de la pratique de la CNDP alors dépourvue de règles de fonctionnement. Le caractère contradictoire des attentes en termes d'idéaux participatif et délibératif, leur décalage avec les pratiques effectives ont bien été soulignés : les " règles du débat » qui commencent à apparaître dans les productions de la CNDP à partir de la seconde moitié des années quatre-vingt-dix, sont marquées par une dualité des référentiels, participatif d'un côté, délibératif de l'autre ${ }^{37}$. La tension entre ces deux référentiels s'éclaire donc si l'on tient compte de la genèse de l'institution, où des chercheurs ont tenté tout à la fois de tester empiriquement certaines propositions inspirées par les travaux d'Habermas, et d'opérationnaliser des règles de la délibération, offrant ainsi à la CNDP une formalisation d'une méthodologie et d'une doctrine qui vont structurer durablement la conduite des réunions publiques par les CPDP.

Ceci explique peut-être $a$ contrario le relatif désintérêt pour les procédures les plus anciennes comme l'enquête publique, trop cadrées par le droit et ne laissant pas place aux chercheurs pour des recommandations d'ordre procédural. La Compagnie des Commissaires Enquêteurs, pour différentes raisons, notamment son ancienneté et son caractère corporatiste, n'a jamais été particulièrement demandeuse auprès de la recherche en sciences sociales pour repenser la procédure, ce type d'expertise revenant à des institutions de premier plan au sein de l'appareil administratif, à commencer par le Conseil d'État.

Il s'agit moins de la construction d'un standard, que de la formalisation de règles déontologiques au sens où elles engagent et permettent d'attester (ou non) la valeur normative des pratiques. C'est ni plus ni moins le caractère démocratiquement vertueux qui est ici en jeu, et que les experts issus du champ académique sont en position d'attester (contrairement à leurs homologues et concurrents consultants).

\section{Militantisme procédural : des plaidoyers en faveur des dispositifs les plus conformes aux attendus de la doctrine délibérative}

L'implication des chercheurs est particulièrement sensible dans un aspect particulier de la montée en puissance de l'industrie de la participation : leur contribution à la normalisation croissante qu'appellent aujourd'hui à la fois l'institutionnalisation et la professionnalisation de la participation. La littérature sur l'évaluation de la concertation est ainsi marquée par un débat sur la question de savoir s'il faut harmoniser, voire normaliser, les pratiques et les dispositifs, ou se contenter de prendre acte de la diversité des formats, des enjeux et des contextes, qui 
exigent des méthodes adaptées. Le cas de la conférence de citoyens, dont on a vu qu'elle était promue comme la procédure la moins éloignée des attendus délibératifs, montre que le militantisme procédural en faveur d'une même technique participative peut revêtir deux formes, selon les acteurs qui la portent. Ainsi, du côté des scientifiques qui cherchent à démocratiser la science et à promouvoir la réflexion éthique, les promoteurs de la conférence de citoyens militent en vue d'une codification de cette technique afin d'empêcher les usages abusifs et les pratiques non contrôlées $^{38}$. Les discours critiques de la technoscience, valorisant les savoirs profanes, ont contribué à valoriser les conférences de citoyens. Mais pour les experts en sciences (sociales) de la participation, le militantisme procédural en faveur de la conférence de citoyens et consorts, passe plutôt par un processus de standardisation, au sens d'une uniformisation des critères d'appréciation de la qualité des procédures, autour d'objectifs - généralement non explicités - de qualité délibérative. De nombreuses propositions en faveur d'une harmonisation des grilles d'évaluation de dispositifs de concertation recèlent ainsi un plaidoyer plus ou moins implicite pour ce type de dispositif ${ }^{39}$.

\section{La structuration et la régulation du marché par un " collège invisible "}

La contribution propre des chercheurs à la construction et la reconfiguration de l'édifice participatif est, sinon déniée, du moins généralement évacuée de l'analyse, et peu perceptible par les acteurs extérieurs à cet univers. Elle prend aujourd'hui la forme d'une entreprise monopolistique permettant de réguler les relations avec les univers sociaux porteurs de la demande, comme avec les professionnels concurrents sur le marché de l'expertise en participation. Le schéma suivant s'appuie sur la cartographie de la communauté épistémique de la concertation au Royaume-Uni, élaborée par Jason Chilvers ${ }^{40}$. Cette cartographie est utilisée ici comme un fond de carte pour représenter le champ des sciences de la participation en France et son rôle de régulation du marché. Le marché des praticiens de la participation (au sens de Nonjon) se trouve dans la partie basse du schéma.

Un ensemble d'acteurs/d'organisations se positionnent aux frontières de ces sous-espaces et fournissent du conseil et de l'aide à la décision au gouvernement et aux grands opérateurs, là où les décideurs n'ont pas déjà développé leurs propres compétences en la matière. Le schéma fait ainsi apparaître à la fois la centralité et la multipositionnalité de certains des acteurs de ce système, qui disposent des ressources leur permettant de se constituer en points de passage obligés. Ce rôle central d'un petit nombre d'acteurs évoque un " collège invisible $»^{41}$, au sens d'un groupe constitué autour d'un front de recherche et dont les membres sont susceptibles de contrôler, aux niveaux local et national, la gestion des fonds de recherche, mais aussi les laboratoires, les ressources symboliques du prestige, et parfois le sort des nouvelles idées scientifiques ainsi que les décisions concernant les stratégies de recherche. Prise dans le sens que lui donne David Demortain, la notion présente un triple intérêt : elle permet de souligner la multiplicité des contextes dans lesquels des chercheurs peuvent agir dès lors qu'ils développent un intérêt pour intervenir 


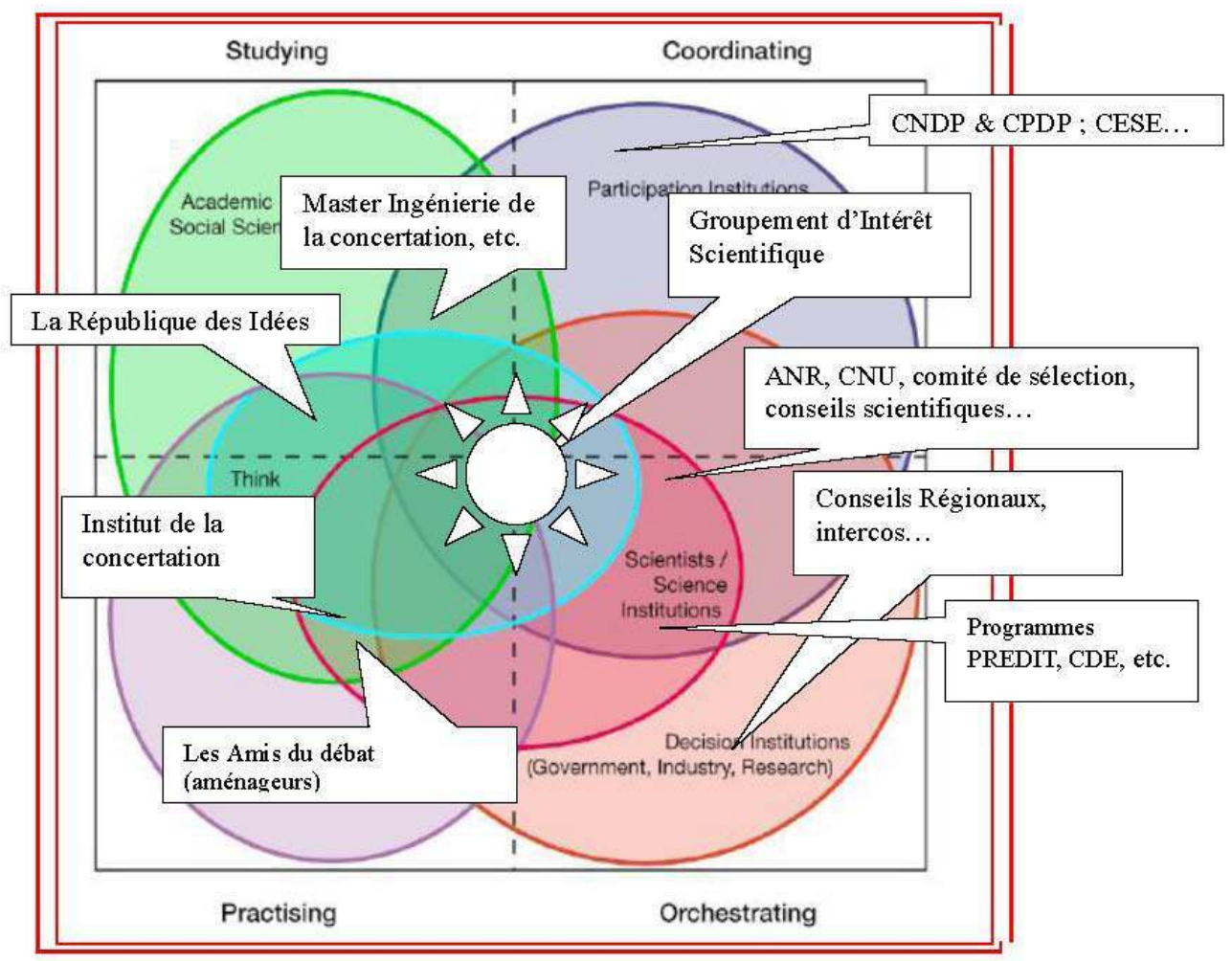

Tableau 2 : le « collège invisible » des sciences de la participation (d'après Chilvers, 2008)

dans la « chose publique », elle met en évidence le caractère relâché de ce réseau rendu invisible car il recouvre la circulation d'un cercle limité d'experts dans des segments distincts. Enfin, elle invite à une analyse sociographique des intérêts, identités et structures sociales de l'expertise.

Le schéma met ainsi en évidence la centralité $\mathrm{du}$ « collège invisible » constitué par le GIS ou sans doute plus précisément de ses initiateurs. De la même manière que pour les notables analysés dans le modèle de la régulation croisée pour comprendre les ressorts du pouvoir local ${ }^{42}$, c'est le cloisonnement entre différents univers sociaux qui leur donne un rôle tout à fait central dans la régulation de ce système. Les différents pôles représentés dans cet espace fonctionnent de façon cloisonnée : ainsi les institutions participatives officielles sont marquées par la fragmentation et l'absence d'articulation. Du côté des prestataires en tout genre, la faible communication tient à une logique de concurrence dans une sphère marchande. Enfin le faible échange d'informations du côté du champ politique tient aux logiques de la compétition politique (électorale) et des phénomènes de 
concurrence entre territoires.

Ce système est à la fois auto-institué, autoréférent et autosuffisant. Il fait exister et « représente » d'une certaine manière, la « demande sociale de participation », à laquelle il s'intéresse et prétend répondre, sans encourir de risque de démenti des faits, puisqu'il n'a pas besoin, pour fonctionner et se perpétuer, d'une participation effective des publics, exclus ou auto-exclus, enrôlés et « parlés » à leur insu.

Ce collège invisible tend à s'instituer en position de régulateur et d'interlocuteur naturel pour toute demande d'expertise ayant trait à la participation, bon nombre de demande, provenant de ou passant par un des organismes partenaires du GIS. Les controverses, désaccords et, démarcations qui peuvent exister entre les acteurs de ce système masquent un accord profond autour d'une vision commune quant au rôle de la science, de sa capacité à nourrir l'action politique, et de la capacité de l'action politique ainsi éclairée à résoudre les problèmes sociaux en proposant toujours plus du même type d'appareillage connu, maîtrisé et socialement valorisé. Les « solutions » apportées et redéfinies indéfiniment dans un tel système sont des solutions techniques, mais cette technique comporte une dimension éminemment politique puisqu'elle repose sur une adhésion profonde à l'idée que le jeu participatif vaut la peine d'être joué. En ce sens, elles constituent les nouvelles technologies sociales de la vertu démocratique ${ }^{43}$.

Tableau 3 : une échelle de la participation des chercheurs à la construction de la démocratie participative

\begin{tabular}{|l|l|}
\hline \multirow{2}{*}{ Collège invisible } & Interlocuteur « naturel » unique ? \\
\cline { 2 - 2 } & Position de régulateur entre différents pôles \\
\hline \multirow{2}{*}{ Militants d'une procédure } & Par la standardisation des évaluations \\
\cline { 2 - 2 } & Par la codification \\
\hline \multirow{2}{*}{ Catalyseurs } & Garantie du respect de la doctrine \\
\cline { 2 - 2 } & Elaboration d'une doctrine \\
\hline \multirow{2}{*}{ Porte-parole d'une demande sociale de papier } & Circulation internationale \\
\cline { 2 - 2 } & Marketing territorial \\
\cline { 2 - 2 } & Mise à l'agenda d'ugenda d'un problème \\
\hline
\end{tabular}




\section{Conclusion : standardisation de la participation et/ou standardisation de la recherche sur la participation ?}

La seconde moitié du vingtième siècle a vu l'émergence en France de la participation comme nouvelle technologie sociale permettant, sinon de construire des choix collectifs, du moins de résoudre (ou de paraître résoudre) un problème de légitimité des décisions et des décideurs. On a pu montrer, en 2000, que la totalisation d'expériences, de pratiques et de procédures hétérogènes était le fait des défenseurs de la « démocratie participative » eux-mêmes, à travers leur activité de « mise en rapport » ${ }^{44}$. C'est ainsi l'action d'un ensemble de réseaux de citoyenneté active qui par son action donne une cohérence à des initiatives hétérogènes, en gommant par un travail de traduction leurs conditions d'apparition et les intérêts variés qu'elles servent, et qui aboutit à la solidification de la notion de démocratie participative. Aujourd'hui, ce sont les « sciences de la participation » qui assurent largement ce travail de mise en rapport, de totalisation et de définition/délimitation des objets et des cadres théoriques légitimes. La recherche contribue de façon active et tangible, bien que souvent déniée, à dessiner les contours et contenus du paysage participatif français et également à en surestimer l'importance sociale. Les chercheurs ne sont pas de simples relais d'une demande qui existerait par ailleurs, mais en sont les vecteurs directs, pour des raisons y compris scientifiques. Si l'écho que ces pratiques rencontrent semble disproportionné par rapport à la réalité qu'elles recouvrent, cette disproportion cesse de surprendre quand on sait le travail d'amplification dont elles font l'objet de la part de tous ceux dont elles servent les intérêts et renforcent la position.

Analysant les modes d'intervention politiques des intellectuels français et leur évolution au $\mathrm{XX}^{\mathrm{e}}$ siècle, Gisèle Sapiro distingue de manière idéal-typique trois facteurs de différenciation :

- La position occupée dans le champ intellectuel selon le volume global du capital symbolique (pôles dominants/dominés).

- L'autonomie par rapport à la demande politique extérieure.

- Le degré de spécialisation de l'activité intellectuelle.

Si l'on trouve représentés, en matière d'expertise de la participation - bien qu'inégalement distribués - tous les modèles d'intervention politiques des intellectuels que Gisèle Sapiro distingue en France, c'est bien l'hétéronomie qui caractérise ce champ de la participation. Les sciences de la participation présentent un caractère militant, qui ne tient pas uniquement au mélange des genres descriptif et prescriptif, largement admis. Il tient aussi au fait que cette recherche est « embarquée » aussi bien dans les logiques de la compétition politique, locale et nationale, que dans les logiques de concurrence propres à la sphère marchande. On peut ainsi penser que par rapport à d'autres objets, la proximité avec l'univers, et les demandes du Politique, est à la fois décisive et largement inaperçue dans cet espace. Le champ de recherche en cours de structuration et de consolidation autour des sciences de la participation se caractérise par une tendance à la spécialisation de plus en plus marquée (revue dédiée) au nom d'un savoir spécialisé, savoir dûment attesté par l'organisation spécifique de la recherche au sein 
d'un réseau thématique de recherche labellisé, qui permet, à défaut de financer des recherches, de réguler la concurrence en matière d'expertise... et sans doute sur le plan académique. Ce champ de recherche est à la fois autonome dans le champ scientifique, et caractérisé par son hétéronomie à l'égard de la demande politique, entendue au double sens de demande proprement politique, et de demande de la part des pouvoirs publics dans le cadre de différentes politiques publiques ${ }^{45}$.

Les sciences de la participation, parce qu'elles sont imbriquées et embarquées dans les champs politiques, administratifs et économiques, en se spécialisant et en se différenciant au sein du champ de la recherche en sciences sociales, tendent aujourd'hui à se construire et à se faire admettre comme les plus légitimes pour énoncer les règles du jeu de la participation. Ce faisant, elles produisent également des effets d'énonciation d'une hiérarchie de la crédibilité des recherches ${ }^{46}$. Cette hiérarchie, qui tend à évacuer de fait plusieurs objets et approches, risque de stériliser doublement la recherche en la matière. En se désintéressant des dispositifs non conformes à ses idéaux, elle risque d'évacuer de l'analyse certains instruments et transformations dont on peut penser qu'ils pèsent infiniment plus sur l'action publique et ses recompositions, que la multiplication des conférences de citoyens et assimilés. C'est également la fonction critique de la recherche qui est questionnée. Ce n'est sans doute pas un hasard si les associations usant des modes d'action les plus radicaux, et les plus critiques à l'égard des dispositifs participatifs ou délibératifs, sont également extrêmement critiques à l'égard de la recherche en sciences sociales sur ces objets ${ }^{47}$.
Il y a donc un besoin nouveau, non seulement de réflexivité critique mais aussi de transparence sur les liens (financiers, de pilotage organisationnel) entre l'organisation de la recherche d'une part, et d'autre part les institutions et les acteurs de l'industrie de la participation. Les orientations privilégiées par toute une génération de jeunes chercheurs, bien représentée dans le présent numéro, nous inclinent à un certain optimisme quant à la possibilité de répondre à ce besoin et ce défi dans les années à venir. 
$\mathrm{N} \cdot \mathrm{O} \cdot \mathrm{T} \cdot \mathrm{E} \cdot \mathrm{S}$

1. Relativement, car de tels travaux existent sur certains dispositifs et plusieurs thèses récentes s'emploient à éclairer cet aspect. Voir G. Gourgues, Le consensus participatif. Les politiques de la démocratie dans quatre régions françaises, thèse de doctorat en science politique, Université de Grenoble, 2010 ; A. Mazeaud, La fabrique de l'alternance. La "démocratie participative » dans la recomposition du territoire régional (Poitou-Charentes 2004-2010), Université La Rochelle, 2010 ; C. Bedu, Quand une citadelle technique se (sou)met à l'épreuve de l'impératif délibératif ». Récit et analyse pragmatique d'une procédure de type «mini-public » dans le domaine de l'eau potable, Thèse de doctorat en science politique, Université de Strasbourg, 2010. Cependant pour différentes raisons leurs conclusions nous paraissent peu exploitées, et n'ont jamais été rassemblées et analysées de façon systématique. Le présent article constitue donc un premier pas dans cette perspective. 2. La « démocratie participative » s'est construite comme le produit de processus sociaux de nature différente, de séries causales largement indépendantes Elle renvoie en effet à des concessions procédurales à l'issue de mouvements sociaux (mai 1968) et de conflits plus limités (les grandes mobilisations antinucléaires des années soixante-dix). Elle renvoie également à des « croisades morales » qui s'appuient, pour justifier leur cause, sur des travaux scientifiques qu'ils contribuent à diffuser et à vulgariser. Elle renvoie enfin aux logiques de la compétition politique qui ont conduit à des réappropriations successives d'un thème qui est toujours apparu après mai 68 , comme politiquement peu risqué et électoralement rentable. L'analyse sociologique des processus ayant conduit, à différents moments de l'histoire de la démocratie représentative, à l'inscription en son sein de nouvelles formes de participation du public, montre ainsi que la mise en place d'un dispositif est rarement le résultat d'une conquête ou même d'une demande citoyenne. La demande sociale supposée fonder et justifier la mise en place de ces dispositifs apparait ainsi comme largement surestimée, et en partie construite par les dispositifs qui prétendent y répondre et par ceux qui les portent. Cf. C. Blatrix, La «démocratie participative», de mai 68 aux mobilisations anti-TGV. Processus de consolidation d'institutions sociales émergentes, thèse de doctorat en science politique, Université Paris 1, 2000, tel.archives-ouvertes.fr/docs/00/72/35/60/PDF/ These_Blatrix.pdf.

3. M. Nonjon, "Quand la démocratie se professionnalise... ", Enquête sur les experts de la participation, Thèse pour le doctorat en science politique, Université Lille 2, 2006.

4. On rejoint ainsi le propos de Yves Déloye, dans sa préface à l'ouvrage dirigé par Catherine Neveu, qui soulignait combien sur cet objet cette porosité entre l'agenda de la recherche et l'agenda de l'action politique devait faire l'objet d'une attention particulière : «Parce qu'elles contribuent à la transformation des dispositifs qu'elles analysent, les sciences sociales du politique (anthropologie, sociologie, science politique...) doivent aussi désormais savoir appréhender cette réflexivité comme faisant partie de leur objet d'investigation. Et ce pour mieux retrouver les conditions de possibilité d'un discours savant autonome $d u$ discours militant mais ouvert au devenir du politique », in C. Neveu (dir.) Cultures et pratiques participatives. Perspectives comparatives, Paris, L'Harmattan, 2007, p. 10. Anthony Giddens a bien souligné comment une forme de réflexivité caractérisait, de façon croissante, les sociétés contemporaines.

5. P. Muller, Le technocrate et le paysan, Paris, les éditions ouvrières, 1984 ; voir aussi dans le domaine 
de l'eau J-P. Le Bourhis, « Complexité et trajectoires d'apprentissage dans l'action publique. Les instruments de gestion durable des ressources en eau en France et au Royaume-Uni », Revue Internationale de Politique Comparée, vol. 10, n², 2003, p.161-175.

6. On pense bien sûr aux pionniers du droit de l'environnement en France, de Michel Prieur à JeanClaude Hélin, René Hostiou, Jacqueline MorandDeviller, Jacques Caillosse...

7. P. Grémion, Le pouvoir périphérique, bureaucrates et notables dans le système politique français, Paris, Éditions du Seuil, 1976, p. 449. P. Grémion élabore cette définition à partir de la distinction entre quatre modèles conflictuels de représentation - démocratie représentative pure, représentation fonctionnelle, démocratie participative, démocratie directe.

8. Voir notamment D. Dulong, Moderniser la politique. Aux origines de la Vé République, Paris, L'Harmattan, 1997.

9. Le droit de la participation qui s'esquisse ainsi est marqué par son caractère épars et fragmenté. Il est ainsi codifié, progressivement et à des époques différentes, dans les codes de l'environnement, de l'urbanisme, des collectivités territoriales, etc. Pour une présentation détaillée des différents textes qui se succèdent à partir du milieu des années quatre-vingt dix. Cf. C. Blatrix, « Concertation et débat public», in O. Borraz, V. Guiraudon (dir.), Politiques publiques, 2. Changer la société, Paris, Presses de Sciences Po, 2010, pp. 213 à 242.

10. M. Ollivier-Trigalo, X. Piechaczyk, Évaluer, débattre ou négocier l'utilité publique, Volet 2 : Le débat public en amont des grands projets d'aménagement : un thème pour une communauté d'idées, collection de l'INRETS, Paris, 2001.

11. Voir sur ce point S. Labranche, P. Warin, $L a$ " concertation dans l'environnement ", ou le besoin de recourir à la recherche en sciences sociales,
Programme CDE, Rapport final, 2006.

12. ADELS, Conseils de quartier, mode d'emploi, 2003 ; Anacej, Comment créer son conseil d'enfants et de jeunes, guide ${ }^{\circ} 2$; D. Bourg, D. Boy, Conférences de citoyens, mode d'emploi, Paris, Charles Léopold Mayer, 2005.

13. M. Paoletti, La démocratie locale et le référendum, Paris, L'Harmattan, 1997.

14. Voir sur ce point H. Nez, J. Talpin, « Généalogies de la démocratie participative en banlieue rouge : un renouvellement du communisme municipal en trompel'œil ? » in Genèses, vol. 2, n79, 2010, pp.79-115.

15. Ce caractère localisé de l'expertise est bien connu dans d'autres sphères, notamment dans la recherche urbaine. Cf. Annales de la recherche urbaine, ${ }^{\circ}{ }^{104}$, 2008.

16. Date de la parution d'un numéro de la revue Politix sur le thème «Démocratie et délibération » qui marque le début de ce « tournant délibératif ».

17. Ainsi, loin de permettre de dépasser le « cens caché » qui caractérise la participation électorale, la démocratie participative tend à creuser l'écart entre une minorité participante qui connaît l'existence de ces dispositifs, et les investit, tout en disposant éventuellement de points d'accès plus directs et moins visibles à la décision, et d'autre part la majorité des citoyens pour laquelle la seule forme de participation reste le vote. Ces conclusions sont confirmées par les travaux récents qui continuent à se pencher sur cette question. Voir A. Mazeaud, J. Talpin, «Participer pour quoi faire? Esquisse d'une sociologie de l'engagement dans les budgets participatifs », Sociologie, vol. 1, numéro 3, 2010, p. 357-374 ; A. Picque, La fabrique de la concertation sur les grands projets. Etudes de cas, Thèse de doctorat en science politique, Université Jules Verne de Picardie, 2012.

18. L. Mermet, "Ouvrir de nouveaux espaces critiques : clarifier, renouveler, «pluraliser» les 
ancrages normatifs des recherches ", in R. Bille et al. (dir.), Concertation, décision, environnement. Regards croisés, Paris, La Documentation Française, 2006, p.81.

19. B. Manin, «L'idée de démocratie délibérative dans la science politique contemporaine. Introduction, généalogie et éléments critiques ", Politix, vol. 15, $\mathrm{n}^{\circ} 57$, pp. 37-55.

20. Voir C. Blatrix « Peut-on évaluer la concertation? Synthèse de la littérature et des débats récents », dans "Qui est vraiment prêt pour évaluer la concertation?» Compte-rendu du séminaire Concertation Décision Environnement du 10 juin 2009. Accessible en ligne : www.concertation-environnement.fr/documents/ seminaires/CDE_Seminaire_Permanent_1.pdf

A. Mazeaud, M-H. Sa Vilas Boas, G.E.K. Berthomé, «Penser les effets de la participation sur l'action publique à partir de ses impensés. Introduction au dossier », Participations, ${ }^{\circ} 1$, 2012, pp. 5-29.

21. M. Callon et al., op. cit., 2001.

22. Pour un plaidoyer en ce sens, questionnant la capacité des dispositifs de concertation à intégrer renforcer la protection de l'environnement, voir L. Mermet, "Concern-Focused Evaluation for Ambiguous and Conflicting Policies. An Appoach from the Environmental Field», American Journal of Evaluation, vol. 31, n², 2010, pp.180-198.

23. Il s'agit là, semble-t-il, d'une spécificité française : de telles analyses existent pourtant dans d'autres contextes nationaux. Voir parmi une littérature abondante, souvent liée à la recherche sur l'aide au développement : C. Boulding, B. Wampler, « Voice, Votes, and Resources: Evaluating the Effect of Participatory Democracy on Well-being ", World Development, vol. 38, n¹, 2010, pp. 125-135.

24. L. Pritchett, M. Woolcok, «Solutions when the Solution is the Problem: Arraying the Disarray in Development », World Development, vol. 32, n²,
2004, pp. 191-212.

25. Voir la convention constitutive du GIS disponible sur son site internet : http://www.participation-etdemocratie.fr.

26. Il est particulièrement difficile - et impossible dans le cadre du présent article - d'aller au-delà d'un simple constat selon lequel certains travaux sont connus et cités, et de donner un sens plus précis à l'idée selon laquelle les connaissances, selon l'expression de A. Giddens, "pénètrent, se dégagent et re-pénètrent les processus sociaux». On peut cependant repérer les analyses et les connaissances qui ont pu, à différentes périodes, inspirer et justifier des entreprises politiques, de telle sorte qu'elles ont contribué, éventuellement « à leur corps défendant ", à la construction de la " démocratie participative ».

27. Dans une démarche proche de celle qu'adopte Franck Poupeau à propos des sciences de l'éducation. Cf. F. Poupeau, Une sociologie d'État. L'école et ses experts en France, Paris, Raisons d'agir, 2003.

28. On reprend ici les trois entrées que François Dudouet, Delphine Mercier et Antoine Vion invitent à articuler pour saisir les dynamiques de normalisation. Cf. F. Dudouet et al. " Politiques internationales de normalisation. Quelques jalons pour la recherche empirique ", Revue Française de Science Politique, vol. 56, n³, 2006, pp. 367-392.

29. Voir la recherche $\mathrm{CDE}$ en cours, ouvrage à paraître début 2013.

30. B. Badura, M. Walltz, « Information behavior in the German federal bureaucracy. The case of the social sciences ", Knowledge, vol. 1, n³, 1980, pp.351-379. 31. L'heuristique de la disponibilité (availability heuristic) est ainsi un phénomène bien connu des psychologues cognitivistes.

32. R. Everett, Diffusions of Innovations, New York, Free Press, 1995, p. 8. C'est nous qui traduisons : « timely, available, and salient »; « The availability 
heuristic suggests that highly visible examples can serve as an information shortcut ».

33. M. Nonjon, op. cit., p. 436.

34. C. Le Bart, L'imputation au maire du développement économique local : L'exemple de trois villes moyennes de l'Ouest intérieur, Thèse de doctorat en science politique, Université de Rennes I, 1989.

35. En France, malgré l'absence de tout cadre juridique précis, une "doctrine » tend à s'imposer, inspirée en partie d'expériences étrangères et en partie par l'expérience de 1998. Le mode d'emploi proposé par D. Bourg et D. Boy formalise ainsi un certain nombre d'adaptations et témoigne d'un processus d'hybridation par rapport au modèle danois. Claire Marris et Pierre-Benoit Joly montrent ainsi comment un certain nombre de règles pratiques dans l'organisation d'une conférence de citoyens ont été formulées à l'occasion de la première expérience française de conférence de citoyens. Cf. C. Marris, P-B. Joly, « Between consensus and citizens: Public participation in Technology Assessment in France », Science Studies, vol. 12, n², 1999, pp. 3-32.

36. G. Mercadal, « La réussite du débat public ouvre la réflexion sur sa portée ", in M. Revel et al., Le débat public : une expérience française de démocratie participative, Paris, La Découverte. 2007, pp. 332-338. 37. B. Reber, « Entre participation et délibération, le débat public et ses analyses sont-ils hybrides du point de vue des théories politiques? », Klesis, vol. $6, n^{\circ} 1$, 2007, pp. 46-78.

38. C'est la position défendue par la Fondation Sciences Citoyennes et par Jacques Testart : voir J. Testart, A. Sinaï, C. Bourgain, Labo Planète ou comment 2030 se prépare sans les citoyens, Paris, 1001 nuits, 2010 ; et le site internet de la Fondation : sciencescitoyennes.org

39. Voir notamment : G. Rowe, L. J. Frewer « Public participation methods : A framework for evaluation »,
Science, Technologie and Human Values, vol. 25, $\mathrm{n}^{\circ} 4$, 2000, p. 3-29 ; M. Callon, P. Lascoumes, Y. Barthe, Agir dans un monde incertain. Essai sur la démocratie technique, Paris, Seuil, 2001 ; D. Bourg, K. Whiteside, Vers une démocratie écologique. Le citoyen, le savant et le politique, Paris, La République des idées, 2010. 40. J. Chilvers, « Environmental risk, uncertainty, and participation: mapping an emergent epistemic community », Environment and Planning A, vol. 40, $\mathrm{n}^{\circ} 12$, 2008, pp. 2990-3008; J. Chilvers, Sustainable Participation? Mapping out and reflecting on the field of public dialogue on science and technology, Harwell, Sciencewise Expert Resource Centre, 2010.

41. D. de Solla Price, Little Science, Big Science, New York, Columbia University Press, 1963; D. Crane, Invisible Colleges. Diffusion of Knowledge in Scientific Communities, Chicago \& London, University of Chicago Press, 1972. Pour un travail récent dans cette perspective voir D. Demortain, Scientists and the Regulation of Risk: Standardising Control, Edward Elgar Publishing, 2011.

42. M. Crozier, J-C. Thoenig, « La régulation des systèmes organisés complexes. Le cas du système de décision politico-administratif local en France », Revue Française de Sociologie, vol. 16, n ${ }^{\circ}$, 1975, pp. 3-32. 43. Expression empruntée à Alain Garrigou dans son analyse socio-historique du suffrage universel.

44. C. Blatrix, op. cit., 2000.

45. Cf. G. Sapiro, « Modèles d'intervention politique des intellectuels. Le cas français », Actes de la Recherche en Sciences Sociales, $\mathrm{n}^{\circ}$ 176-177, pp. 8-31. 46. H. Becker, Les ficelles du métier. Comment conduire sa recherche en sciences sociales, Paris, La Découverte, 2002, p. 153.

47. C'est le cas de l'association Pièces et Main d'œuvres, qui milite contre les nanotechnologies et dénonce régulièrement le rôle de caution que joue la recherche en sciences sociales. 


\title{
$R \cdot E ́ \cdot S \cdot U \cdot M \cdot E ́$
}

L'article est centré sur un angle mort de l'histoire sociale de la démocratie participative, à savoir la contribution spécifique des chercheurs à la construction et la consolidation de l'édifice participatif français. Cette contribution propre est analysée ici en combinant une approche diachronique et un point de vue synchronique sur ce que l'on propose d'appeler les sciences de la participation. Le paysage participatif français a évolué dans le temps et s'est reconfiguré en intégrant partiellement les connaissances produites par les sciences sociales sur les dispositifs et leur fonctionnement. L'analyse de ces reconfigurations, et des formes variées que peut prendre l'implication des chercheurs, permet de comprendre pourquoi la contribution propre du champ scientifique au développement de la participation du public en France est à la fois centrale, et largement occultée.

\begin{abstract}
This article is focused on a dead angle in the social history of participatory democracy, that is the specific contribution of the academics to the construction and consolidation of the French participatory framework. Combining a diachronic and a synchronic point of view, the very contribution of the academic field is analyzed through the term of "participation studies". Over time, the French participatory landscape has been modified and redefined through the partial incorporation of knowledge produced by social sciences on the participatory devices and their functioning. The analysis of these evolutions and of the diverse forms of scholars' implication, allows us to understand the reasons why the role of academics is both crucial, and largely eclipsed.
\end{abstract}

